\title{
On the role of the local oscillator intensity in optical homodyne-like tomography
}

\author{
Stefano Olivares \\ Quantum Technology Lab, Dipartimento di "Fisica Aldo Pontremoli", \\ Università degli Studi di Milano, and INFN, Sezione di Milano, I-20133 Milano (Italia) \\ Alessia Allevi* \\ Dipartimento di Scienza e Alta Tecnologia, \\ Università degli Studi dell'Insubria, I-22100 Como (Italia) \\ Maria Bondani \\ Istituto di Fotonica e Nanotecnologie, CNR, I-22100 Como (Italy)
}

\begin{abstract}
In a recent research (S. Olivares, et al., New J. Phys. 21 (2019) 103045) we have demonstrated that a homodyne-like scheme, exploiting a "low-intensity" local oscillator, can be used to perform optical state tomography of both quantum and classical states of light. The reconstruction method directly uses the homodyne-like probability distribution retrieved from the detector. Here, we further investigate the role played by the local oscillator in this respect. In particular, we study to some extent how its intensity affects the quantum-state reconstruction procedure by focusing on the case of the Fock states $|1\rangle$ and $|2\rangle$, whose homodyne-like probability distributions are sensibly affected by the actual value of the LO intensity. The analysis is performed on Monte Carlo simulated experiments taking also into account the quantum detection efficiency.

Keywords: Quantum tomography, Fock states, photon-number-resolving detectors, homodyne-like detector
\end{abstract}

\footnotetext{
* Corresponding author

Email addresses: stefano.olivares@fisica.unimi.it (Stefano Olivares), alessia.allevi@uninsubria.it (Alessia Allevi), maria.bondani@uninsubria.it (Maria Bondani)
}

Preprint submitted to Journal of ${ }^{A} T_{E} X$ Templates

March 19, 2020 


\section{Introduction}

The development of quantum technologies requires quantum resources that can be practically exploited. Therefore, the full characterization of the quantum states to be employed represents an unavoidable step. Among the tech5 niques proposed in the optical domain, homodyne tomography is one of the most used, giving it access to the full description of a quantum state in terms of Wigner function and density matrix 1, 2, From the experimental point of view, quantum-state tomography is based on measurements performed with a homodyne detector, namely an interferometric scheme in which the signal state under examination is mixed with a second field, called local oscillator (LO), at a balanced beam splitter (BS). In the traditional scheme, the LO is a macroscopic coherent field that can be described classically. The two outputs of the intereferometer are detected by two p-i-n photodiodes, whose difference photocurrent is formed, amplified, rescaled by the LO amplitude and recorded as a function of the LO phase. The application of suitable reconstruction methods to the data from homodyne detection allows the full characterization of the signal state. Among them, the most used are maximum-likelihood methods [3, 4, 5, 6, 7, , 8, and algorithms based on the so-called pattern functions [9, 10, 11, 12, 13, 14, 15].

We have recently proved that quantum-state tomography can be achieved also with a homodyne-like scheme, in which a low-intensity LO and photonnumber-resolving detectors are used instead of a macroscopic LO and p-i-n photodiodes. Moreover, the possibility to develop homodyne detection strategies using e.g multiplexed schemes has been discussed also in [16] and [17].

In Ref. [18 we have shown the experimental reconstruction of classical states, such as coherent states and phase-averaged coherent states, obtained by means of hybrid photodetectors, which are commercial detectors endowed with partial photon-number resolution and a linear response up to 100 photons. One main limitation of this class of detectors is the quantum efficiency, which is roughly $50 \%$ in the green spectral region and can be further reduced in a realistic setup. A valid alternative, especially for what concerns the high value of the 
quantum efficiency, is represented by transition-edge sensors (TES), and some homodyne-like detection schemes employing them have been very recently published [19, 20]. Nevertheless, one of the main limitations of TES is given by their dynamic range, which is approximately equal to 10 detected photons. Thus, TES cannot be used to investigate the so-called mesoscopic intensity domain, in which the intensity of the LO can reach values as large as 50 photons. On the contrary, the most promising photon-number-resolving detectors that can be operated over a wide dynamic range are Silicon photomultipliers (SiPMs). They are essentially a matrix of avalanche diodes, called cells, connected in parallel to a single output. Each diode is reverse-biased at a voltage value exceeding the breakdown threshold, and it works in Geiger Müller regime, yielding a standard output signal at any detection event. Assuming that at most one photon impinges on a single cell, the number of fired cells gives the number of incident photons. Unfortunately, these detectors are affected by some spurious 45 effects, such as dark counts, cross talk and afterpulses. Moreover, the current value of their quantum efficiency is up to $60 \%$. In the context of quantumstate tomography, such drawbacks unavoidably affect the reconstructed state. Nevertheless, we notice that SiPMs response can be suitably modelled [21] and, by properly acting on the control parameters, such as the bias voltage and the integration gate, it is possible to substantially reduce all the spurious effects [21, 22]. These parameters also control the photon-number-resolving capability of SiPMs, that is the ability to distinguish among one, two, three or more photons. For instance, reducing the integration gate can improve the separation among peaks, thus enhancing the determination of the statistical properties of light. Thanks to the optimized operation of SiPMs, we have recently proved that such detectors can be exploited to test both nonclassical correlations and subPoissonianity of optical states 22$]$.

In this paper we investigate the effects on the reconstruction procedure of varying the intensity of the LO. In Ref. [18] we have demonstrated that the 60 scheme can be suitable for the reconstruction of both classical and nonclassical states of light (such as cat states), upon optimization of the value of the LO 
with respect to the signal state. The results obtained in Ref. [18] suggest that the intensity of the LO must be increased not only when the energy of the reconstructed states increases, but also when the states exhibit peculiar Wigner functions with, for instance, many oscillations in the phase space. In this last scenario, increasing the LO intensity allows a better sampling of the corresponding "discretized" homodyne probability distributions and, thus, a more faithful tomographic reconstruction. Here, we aim at studying the effect of the intensity of the LO on the fidelity of the tomographic reconstruction and we consider the paradigmatic cases of the Fock states $|1\rangle$ and $|2\rangle$, which show an increasing number of oscillations of the homodyne probability distributions, both in the case of a perfect detection efficiency and in the non ideal one.

\section{Materials and methods}

The standard homodyne scheme mentioned in the Introduction allows to retrieve the information about the quadrature operators $\hat{x}_{\phi}$ of the signal state by recording the difference photocurrent, described by the operator, $\Delta \hat{I}$, between the two BS outputs. Here, $\phi$ is the relative phase between the signal and the LO, which is assumed to be a highly excited classical coherent field. If $\beta$ is the complex amplitude of the LO and $\hat{a}$ and $\hat{a}^{\dagger}$, with $\left[\hat{a}, \hat{a}^{\dagger}\right]=1$, are the annihilation and creation operators of the signal field, respectively, one can show that

$$
\lim _{|\beta| \rightarrow \infty}\left(\frac{\Delta \hat{I}}{\sqrt{2}|\beta|}\right)^{n}=\left(\frac{\hat{a}^{\dagger} e^{i \phi}+\hat{a} e^{-i \phi}}{\sqrt{2}}\right)^{n} \equiv \hat{x}_{\phi}^{n},
$$

where $n \in \mathbb{N}$.

In the case of homodyne-like detection, the physical quantity which we have access to is given by the photon-number difference $\Delta=m-n$ between the two outputs of the detectors, whose probability distribution $p_{\mathrm{D}}(\Delta, \phi)$ reduces to the standard homodyne one $p_{\mathrm{HD}}(x, \phi)$ when the LO becomes intense [23, 19, 20, namely,

$$
p_{\mathrm{D}}(\Delta, \phi) \rightarrow p_{\mathrm{HD}}(x=\Delta /(\sqrt{2}|\beta|), \phi) /(\sqrt{2}|\beta|),
$$


where $|\beta|$ is the amplitude of the LO described by a coherent state with complex amplitude $\beta=|\beta| e^{i \phi}$. In this case, one finds

$$
\left(\frac{\Delta \hat{I}}{\sqrt{2}|\beta|}\right)^{n}=\hat{x}_{\phi}^{n}+\frac{\gamma_{\phi}^{(n)}\left(\hat{a}, \hat{a}^{\dagger}\right)}{|\beta|^{2}},
$$

${ }_{75} \gamma_{\phi}^{(n)}\left(\hat{a}, \hat{a}^{\dagger}\right)$ being a suitable function of the annihilation and creation operators which can be obtained performing direct calculations. For instance, the first three terms are $\gamma_{\phi}^{(1)}\left(\hat{a}, \hat{a}^{\dagger}\right)=0, \gamma_{\phi}^{(2)}\left(\hat{a}, \hat{a}^{\dagger}\right)=\hat{a}^{\dagger} \hat{a}$, and $\gamma_{\phi}^{(3)}\left(\hat{a}, \hat{a}^{\dagger}\right)=3 \hat{a}^{\dagger} \hat{x}_{\phi} \hat{a}+\hat{x}_{\phi}$. The last term in Eq. $(3)$ scales as $1 /|\beta|^{2}$, where $|\beta|^{2}$ is the energy of the LO, and it vanishes in the limit $|\beta|^{2} \gg 1$, as one may expect: this is the standard homodyne detection working regime. However, in the presence of a low intensity LO, the function $\gamma_{\phi}^{(n)}\left(\hat{a}, \hat{a}^{\dagger}\right)$ in Eq. 33 can become relevant for the tomographic reconstruction method based on the homodyne-like probabilities, since it affects their moments and, thus, also the moments of the reconstructed quadratures. Therefore, the contribution of $\gamma_{\phi}^{(n)}\left(\hat{a}, \hat{a}^{\dagger}\right)$, whose actual expectation value is state-dependent, should be made negligible by properly choosing the LO intensity in the homodyne-like detection scheme.

In Ref. [18, we have demonstrated that the probability distribution for the photon-number difference can be written in terms of the joint photon-number statistics $q(m, n)$ measured at the two BS outputs:

$$
p_{\mathrm{D}}(\Delta, \phi)=\sum_{m, n: m-n=\Delta} q(m, n),
$$

in which 24,25$]$

$$
q(m, n)=\int_{\mathbb{C}} d^{2} \zeta P(\zeta) e^{-\mu_{c}(\zeta, \beta)-\mu_{d}(\zeta, \beta)} \frac{\left[\mu_{c}(\zeta, \beta)\right]^{n}\left[\mu_{d}(\zeta, \beta)\right]^{m}}{n ! m !} .
$$

In this expression, $P(\zeta)$ is the Glauber-Sudarshan $P$-representation of the generic signal state $\rho=\int_{\mathbb{C}} d^{2} \zeta P(\zeta)|\zeta\rangle\langle\zeta|$, whereas $\mu_{c}(\zeta, \beta)=|\beta+\zeta|^{2} / 2$ and $\mu_{d}(\zeta, \beta)=$ $|\beta-\zeta|^{2} / 2$ are the mean values of the distributions of the two BS output modes $90 \quad c$ and $d$. In the case of a non-ideal detection, the effect of a quantum efficiency $\eta_{k} \neq 0$ ( $k$, being equal to $\left.c, d\right)$ on the output modes may be taken into account by replacing $\mu_{k}(\zeta, \beta)$ with $\eta_{k} \mu_{k}(\zeta, \beta)$. 
Here, we consider the reconstruction of the Fock state $\rho=|\nu\rangle\langle\nu|, \nu \in \mathbb{N}$, whose Glauber-Sudarshan $P$-representation is given by

$$
P(\zeta)=\sum_{m=0}^{\nu}\left(\begin{array}{c}
\nu \\
m
\end{array}\right) \frac{1}{m !}\left(\frac{\partial^{2}}{\partial \zeta \partial \zeta^{*}}\right)^{m} \delta^{2}(\zeta)
$$

for which the joint photon-number distribution reads as

$$
\begin{aligned}
q(m, n)=\frac{e^{-|\beta|^{2} / 2}}{\sqrt{\nu !}} & {\left[\sum_{k=0}^{\nu}\left(\begin{array}{l}
\nu \\
k
\end{array}\right) \sqrt{\left(\begin{array}{c}
n+m-\nu \\
m-k
\end{array}\right)} \frac{(-1)^{n-\nu+k}}{\sqrt{2^{n+m}}}\right.} \\
& \left.\times \frac{\beta^{n+m-\nu} \sqrt{n ! m !}}{\sqrt{(m-k) !(n-\nu+k) !(n+m-\nu) !}}\right]^{2} .
\end{aligned}
$$

An analogous expression of $q(n, m)$ can be written in the case of a non-ideal detection efficiency. Since it is quite clumsy, we decided not to show it here.

As already demonstrated in Ref. [18, the knowledge of the homodyne-like distribution can be exploited to calculate the elements $\rho_{n m}$ of the density matrix of the Fock states. By following the methods proposed by Leonhardt et al. in [9], for a homodyne-like detection we have

$$
\rho_{n m}=\int_{0}^{\pi} d \phi \int_{-n_{\max }}^{+n_{\max }} d \Delta p_{\mathrm{D}}(\Delta, \phi) F_{n m}(\Delta, \phi) .
$$

The functions $F_{n m}(\Delta, \phi)$ are a set of sampling functions written as 9 ]

$$
F_{n m}(\Delta, \theta)=f_{n m}(\Delta) \exp [i(n-m) \theta],
$$

where the pattern functions $f_{n m}(\Delta)$ can be expressed in terms of regular and irregular wave functions, $\psi_{n}(\Delta)$ and $\phi_{m}(\Delta)$, respectively:

$$
\begin{aligned}
f_{n m}(\Delta)=2 x \psi_{n}(\Delta) \phi_{m}(\Delta)-\sqrt{2(n+1)} & \psi_{n+1}(\Delta) \phi_{m}(\Delta) \\
& -\sqrt{2(m+1)} \psi_{n}(\Delta) \phi_{m+1}(\Delta),
\end{aligned}
$$

with $m \geq n$. Quite simple expressions for the numerical computation of these functions can be found in the original paper [9].

\section{Results}

\subsection{Single-photon Fock states}

The analysis we present in the following concerns the Fock states $|1\rangle$ and $|2\rangle$ and is based on Monte Carlo simulated experiments. In order to properly 

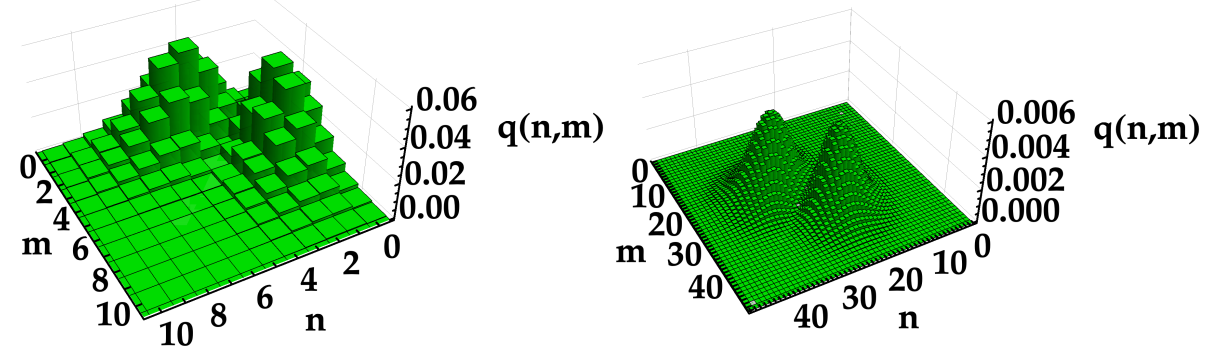

Figure 1: Joint-photon-number distribution in the case of Fock state $|1\rangle$ for $\eta=1$ and different choices of the LO intensity. Left: $|\beta|^{2}=5$, Right: $|\beta|^{2}=50$. Note that the different ranges in the three axes of the two panels are due to the fact that the joint-photon-number distributions are built with two different choices of LO.
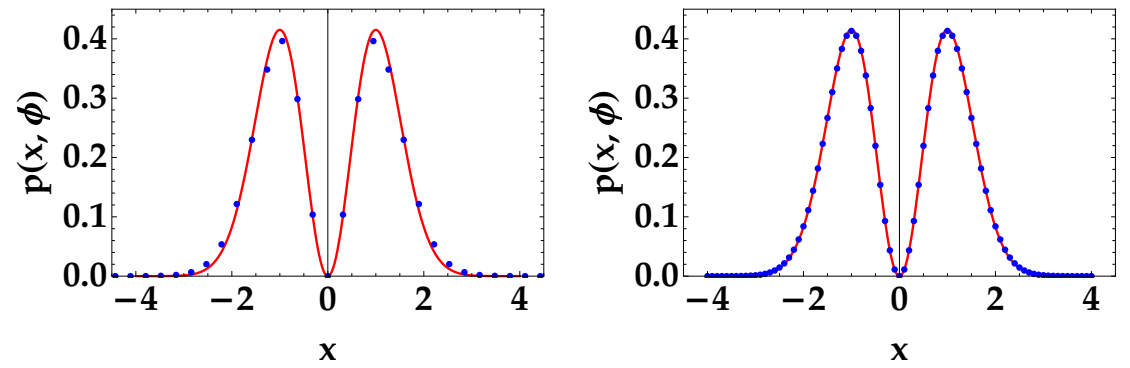

Figure 2: Homodyne-like distribution $p_{\mathrm{D}}$ in the case of Fock state $|1\rangle$ for $\eta=1$ and different choices of the LO intensity (blue points). Left: $|\beta|^{2}=5$, Right: $|\beta|^{2}=50$. In each panel, the theoretical homodyne distribution is shown as red solid line. The fidelity between the two curves is equal to $99.612 \%$ for $\left|\beta^{2}\right|=5$ and $99.999 \%$ for $\left|\beta^{2}\right|=50$. In order to compare $p_{\mathrm{D}}$ and $p_{\mathrm{HD}}$, we suitably rescaled the photon-number difference $\Delta$ appearing in Eq. (4).

evaluate the statistical errors in the reconstruction procedure, for each state we repeat the simulation 10 times and use 30,000 data in each run. First of all, we consider the state $|1\rangle$ with $\eta=1$ and $\eta<1$ (for the sake of simplicity we assume that both the detectors have the same quantum efficiency $\eta$ ). To investigate the role played by the intensity of the LO in the reconstruction of the state, we consider six possible intensity values, namely $|\beta|^{2}=5,10,20,30$, 40, and 50. In Fig. 1. we show, for the two extreme values, the joint photonnumber distribution, which exhibits a double-peak structure, as expected. Note that, the larger the value of $|\beta|^{2}$ the better the distinction between the two peaks and the "resolution" of the distribution. The corresponding homodyne- 

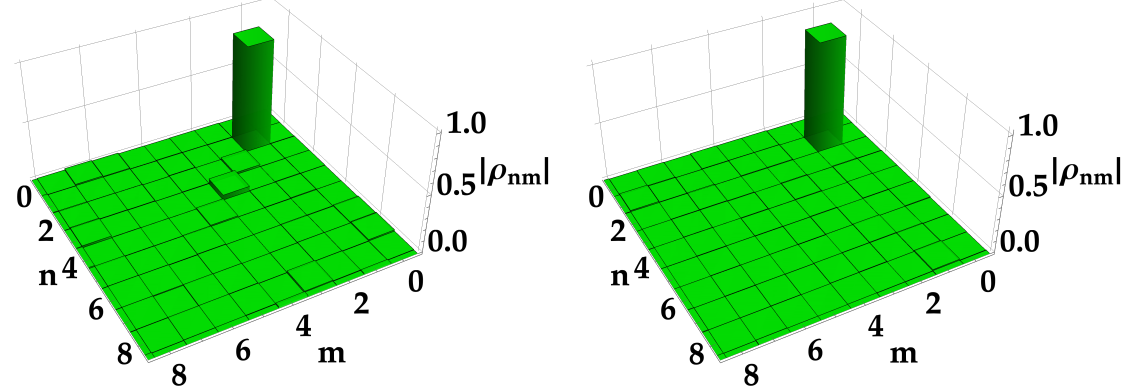

Figure 3: Reconstruction of the density matrix in the case of Fock state $|1\rangle$ for $\eta=1$ and different choices of the LO intensity. Left: $|\beta|^{2}=5$, Right: $|\beta|^{2}=50$.

like distributions, obtained from Eq. (4) for the joint-probability distributions in Eq. (7), are shown in the panels of Fig. 2 for $|\beta|^{2}=5$ and $|\beta|^{2}=50$, together with the theoretical homodyne probability distributions

$$
p_{\mathrm{HD}}(x, \phi)=\frac{1}{\sqrt{2}} e^{-x^{2}} \sqrt{\frac{2}{\pi}} 2 x^{2} .
$$

By comparing the homodyne-like distributions with the corresponding homodyne ones, we can clearly see that the larger the intensity $|\beta|^{2}$, the better the superposition. In general, it is interesting to notice that the two distributions are well superimposed even for a small imbalance between signal and LO. Indeed, for the values of $|\beta|^{2}$ of Fig. 2, the fidelities $f_{\mathrm{H}}=\sum_{i=1}^{N} \sqrt{p_{i, \mathrm{D}} p_{i, \mathrm{HD}}}$ between the two distributions are equal to $99.612 \%$ and $99.992 \%$, respectively.

The slight discrepancy between homodyne and homodyne-like distributions does not prevent the reconstruction of the density matrix according to the method presented in [9]. However, the direct observation of the obtained matrices in Fig. 3 witnesses the limitations imposed by a low-intensity LO. Indeed, in the left panel, corresponding to the case in which $|\beta|^{2}=5$, the density matrix exhibits a peak at $n=1$ which is lower than 1 . On the contrary, in the right panel, corresponding to the case in which $|\beta|^{2}=50$, the reconstruction is definitely better. Note that, the maximum error on a single element of the density matrix is equal to 0.014 for $|\beta|^{2}=5$ and 0.009 for $|\beta|^{2}=50$, whereas the mean error on a single reconstructed element is equal to 0.006 for $|\beta|^{2}=5$ and 0.004 for $|\beta|^{2}=50$. 


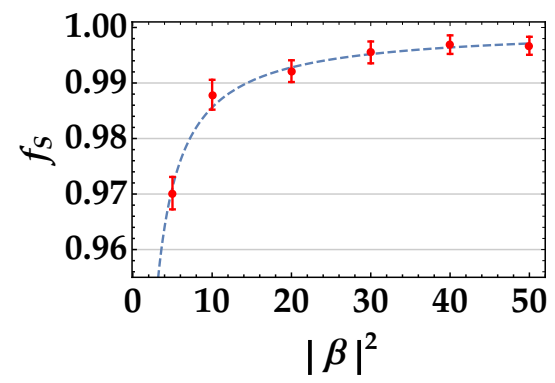

Figure 4: State fidelity in the case of Fock state $|1\rangle$ as a function of the LO intensity for $\eta=1$. The dashed line corresponds to the fitting function $g_{a}\left(|\beta|^{2}\right)$ with $a=0.144 \pm 0.008$.

Since the Fock states are diagonal, the state fidelity can be evaluated as $f_{\mathrm{S}}=$ $\sum_{m=0}^{\nu} \sqrt{\rho_{\mathrm{mm}, \exp } \rho_{\mathrm{mm}, \mathrm{th}}}$. For the two cases shown in Fig. $3, f_{\mathrm{S}}$ is equal to $97.02 \pm 0.29 \%$ and $99.67 \pm 0.16 \%$, respectively. We note that, in the quantum regime, only very high values of the fidelities can be considered acceptable when dealing with the reliable generation of a required quantum state [26. In order to better emphasize the dependence of fidelity on the intensity of the LO, in Fig. 4 we plot $f_{\mathrm{S}}$ as a function of $|\beta|^{2}$, where the saturation at 1 at increasing intensity values of the LO is clearly visible. The data can be numerically fitted by the function

$$
g_{a}\left(|\beta|^{2}\right)=1-\frac{a}{|\beta|^{2}}
$$

where $a$ is a real fitting parameter. It is worth noting that the scaling $1 /|\beta|^{2}$ appearing in $g_{a}\left(|\beta|^{2}\right)$ is consistent with the scaling of the additional term in Eq. (3): in the limit $|\beta|^{2} \gg 1$, the results of the homodyne-like detection approaches those corresponding to the standard homodyne one.

When $\eta<1$, the reconstructed state is no longer that expected in the ideal case, i.e. for $\eta=1$. For instance, in the case of the Fock state $|1\rangle$, it has been demonstrated, theoretically, numerically and experimentally, that its density matrix displays two terms on the diagonal, namely a peak corresponding to 0 photon in addition to that corresponding to 1 photon [27. More in general, in the case of the Fock state $|\nu\rangle$, the reconstructed density matrix [28]

$$
\rho=\sum_{\nu=0}^{n}\left(\begin{array}{l}
n \\
\nu
\end{array}\right) \eta^{\nu}(1-\eta)^{n-\nu}|\nu\rangle\langle\nu|
$$



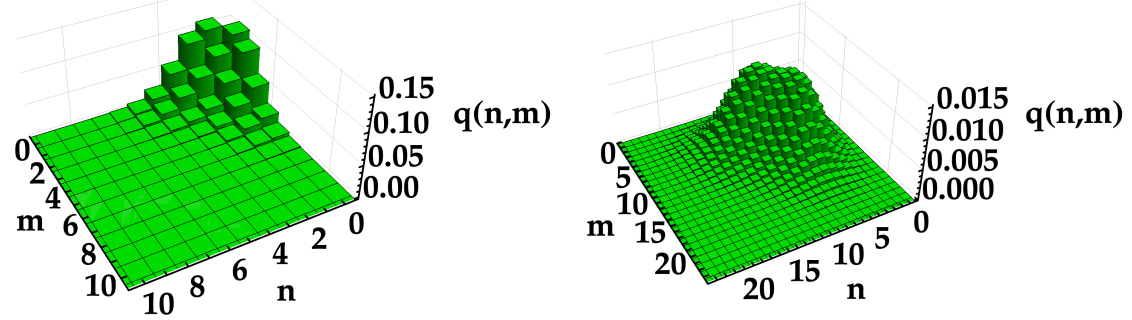

Figure 5: Joint-photon-number distribution in the case of Fock state $|1\rangle$ for $\eta=0.4$ and different choices of the LO intensity. Left: $|\beta|^{2}=5$, Right: $|\beta|^{2}=50$.
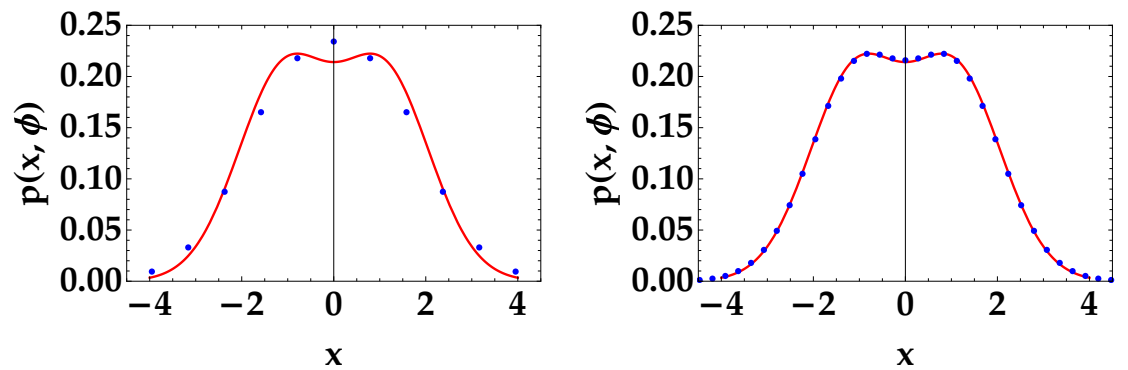

Figure 6: Homodyne-like distribution $p_{\mathrm{D}}$ in the case of Fock state $|1\rangle$ for $\eta=0.4$ and different choices of the LO intensity (blue points). Left: $|\beta|^{2}=5$, Right: $|\beta|^{2}=50$. In each panel, the theoretical homodyne distribution is shown as red solid line. The fidelity between the two curves is equal to $99.599 \%$ for $|\beta|^{2}=5$ and $99.947 \%$ for $|\beta|^{2}=50$. In order to compare $p_{\mathrm{D}}$ and $p_{\mathrm{HD}}$, we suitably rescaled the photon-number difference $\Delta$ appearing in Eq. 44.

is given by $n+1$ contributions. In the following we present the results achieved for the Fock states $|1\rangle$ in the case $\eta=0.4$, which is a reliable value of the detection efficiency of commercial photon-number-resolving detectors.

In Fig. 5 , we plot the joint probability distributions for the extreme values of the LO, namely, $|\beta|^{2}=5$ and $|\beta|^{2}=50$. Note that, due to the low quantum efficiency, it is no more possible to distinguish the two peaks shown in the analogous Fig. 1. The same result holds for the corresponding homodyne-like distributions in Fig. 6. Indeed, instead of having a good separation between the peaks, we have a unique large structure with only a small dip on the top. Moreover, it is well evident that the higher the LO intensity the better the superposition to the standard homodyne distribution. On the other hand, the weaker the $\mathrm{LO}$, the higher the contribution to $p_{\mathrm{D}}(x, \phi)$ at $x=0$ : This is clear 

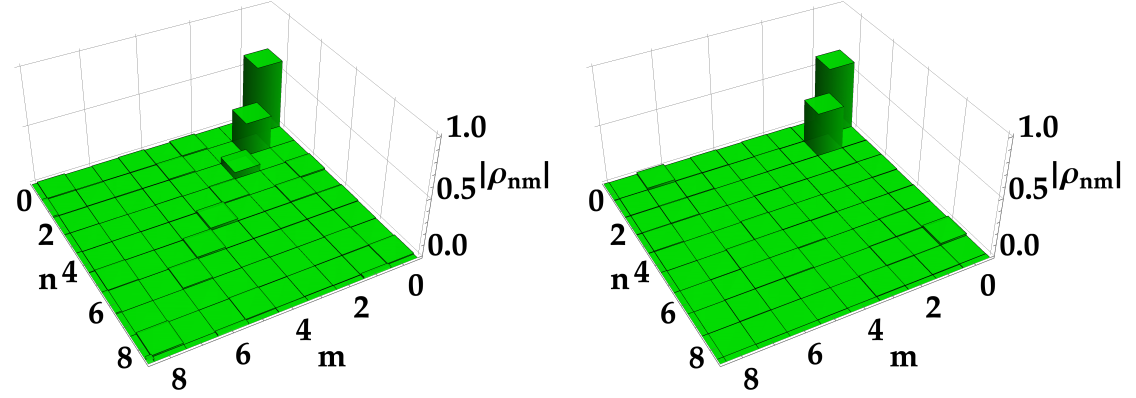

Figure 7: Reconstruction of the density matrix in the case of Fock state $|1\rangle$ for $\eta=0.4$ and different choices of the LO intensity. Left: $|\beta|^{2}=5$, Right: $|\beta|^{2}=50$.

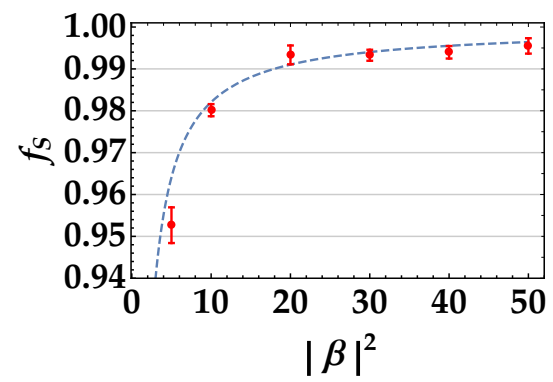

Figure 8: State fidelity in the case of Fock state $|1\rangle$ as a function of the LO intensity for $\eta=0.4$. The dashed line corresponds to the fitting function $g_{a}\left(|\beta|^{2}\right)$ with $a=0.224 \pm 0.017$.

by inspecting the plot with $|\beta|^{2}=5$ in Fig. 6

The application of the reconstruction method to the case under examination yields to the results shown in Fig. 7 where the presence of the vacuum contribution, $\rho_{00}$, is clearly visible. We notice that for $|\beta|^{2}=5$ also the peak corresponding to $\rho_{22}$ on the diagonal is visible. This depends on the fact that, when the signal and the LO are similar, the assumption that the LO can be classically treated does not hold anymore and thus the reconstructed state contains information both on the signal and on the LO. Here, the maximum error on a single element of the density matrix is equal to 0.019 for $|\beta|^{2}=5$ and 0.012 130 for $|\beta|^{2}=50$, whereas the mean error on a single reconstructed element is equal to 0.006 for $|\beta|^{2}=5$ and 0.005 for $|\beta|^{2}=50$. In this case, the fidelities to the expected states are equal to $95.27 \pm 0.43 \%$ for $|\beta|^{2}=5$ and to $99.55 \pm 0.18 \%$ for $|\beta|^{2}=50$. As also proved by the values of fidelity reported in Fig. 8 , the larger 

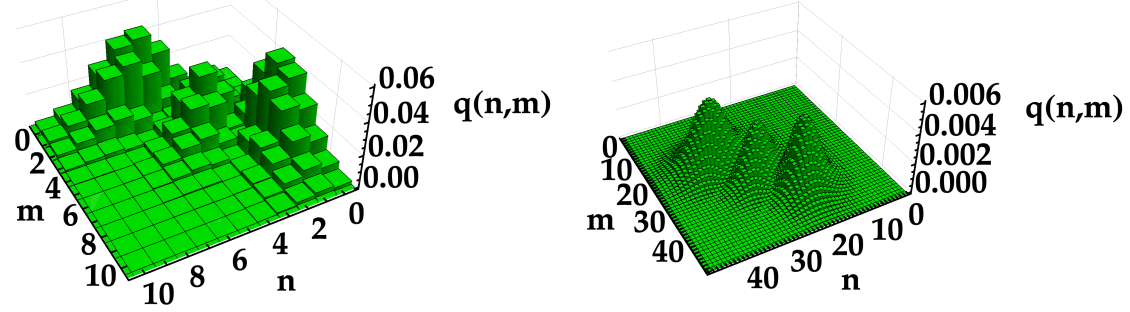

Figure 9: Joint-photon-number distribution in the case of Fock state $|2\rangle$ for $\eta=1$ and different choices of the LO intensity. Left: $|\beta|^{2}=5$, Right: $|\beta|^{2}=50$.

the LO the better the reconstruction. We also note that, compared to the case with $\eta=1$, the values of fidelity are slightly worse. This is due to the fact that not only the signal is affected by a low quantum efficiency, but also the intensity of the LO, which is now reduced by a factor $\eta$.

\subsection{Two-photon Fock states}

The results achieved so far give proof of the possibility to fully characterize a single-photon Fock state even in the presence of a low-intensity LO. This can be obtained both in the ideal case and in the presence of an imperfect detection.

In this Section we want to explore the situation of more populated Fock states, namely two-photon Fock states. First of all, we consider a Fock state $|2\rangle$ for $\eta=1$ and compare the results with those achieved in the previous Section. We consider the same values of $|\beta|^{2}$, namely $5,10,20,30,40$, and 50. In Fig. 9 we show the joint probability distribution for the two extreme cases.

The distributions exhibit three peaks, which become more separated at increasing intensity values of the LO. The same holds for the homodyne-like distributions shown in Fig. 10. Here, for $|\beta|^{2}=5$, it is also quite evident the discrepancy between the homodyne-like and the homodyne distributions, which in this case reads as

$$
p_{\mathrm{HD}}(x, \phi)=e^{-x^{2}} \sqrt{\frac{1}{\pi}} \frac{H_{2}(x)}{8},
$$

$H_{\nu}(x)$ being the Hermite polynomial with $\nu=2$. 

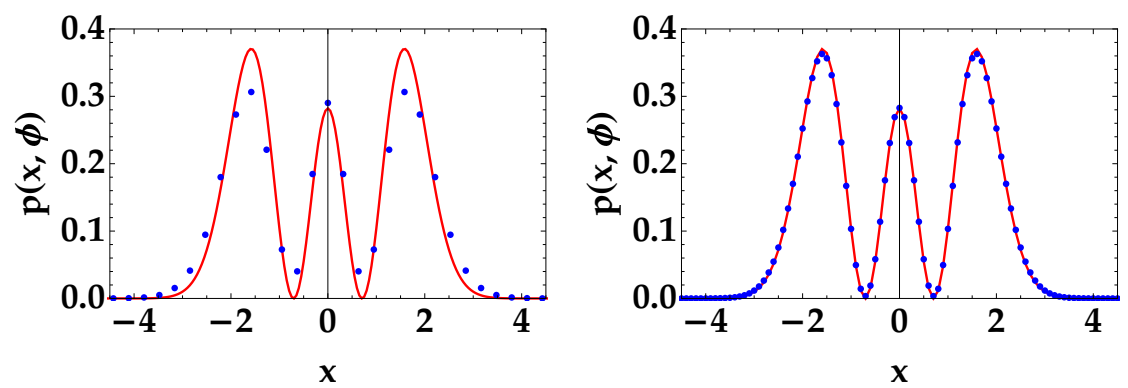

Figure 10: Homodyne-like distribution $p_{\mathrm{D}}$ in the case of Fock state $|2\rangle$ for $\eta=1$ and different choices of the LO intensity (blue dots). Left: $|\beta|^{2}=5$, Right: $|\beta|^{2}=50$. In each panel, the theoretical homodyne distribution is shown as red solid line. The fidelity between the two curves is equal to $98.525 \%$ for $|\beta|^{2}=5$ and $99.962 \%$ for $|\beta|^{2}=50$. In order to compare $p_{\mathrm{D}}$ and $p_{\mathrm{HD}}$, we suitably rescaled the photon-number difference $\Delta$ appearing in Eq. (4).
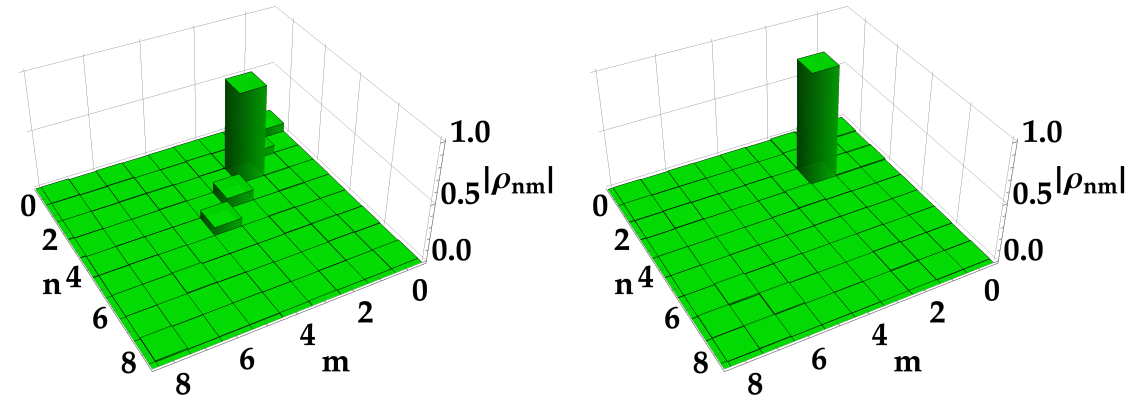

Figure 11: Reconstruction of the density matrix in the case of Fock state $|2\rangle$ for $\eta=1$ and different choices of the LO intensity. Left: $|\beta|^{2}=5$, Right: $|\beta|^{2}=50$.

Concerning the reconstruction of the density matrix, the corresponding plots are shown in Fig. 11 in the case $|\beta|^{2}=5$ the density matrix is not very well reconstructed due to the presence of diagonal elements other than 2 , while in the case $|\beta|^{2}=50$ it is properly retrieved. Note that, the maximum error on a single element of the density matrix is equal to 0.010 for $|\beta|^{2}=5$ and 0.010 for $|\beta|^{2}=50$, whereas the mean error on a single reconstructed element is equal to 0.005 for $|\beta|^{2}=5$ and 0.004 for $|\beta|^{2}=50$. The fidelities to the expected states are equal to $89.93 \pm 0.42 \%$ for $|\beta|^{2}=5$ and to $98.76 \pm 0.14 \%$ for $|\beta|^{2}=50$. The fidelity between each reconstructed matrix and the corresponding theoretical one as a function of the LO intensity is highlighted in Fig. 12.

As the last case, we consider the reconstruction of the Fock state $|2\rangle$ for 


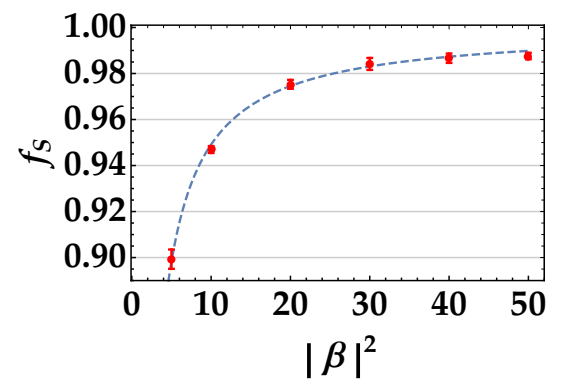

Figure 12: State fidelity in the case of Fock state $|2\rangle$ as a function of the LO intensity for $\eta=1$. The dashed line corresponds to the fitting function $g_{a}\left(|\beta|^{2}\right)$ with $a=0.508 \pm 0.017$.
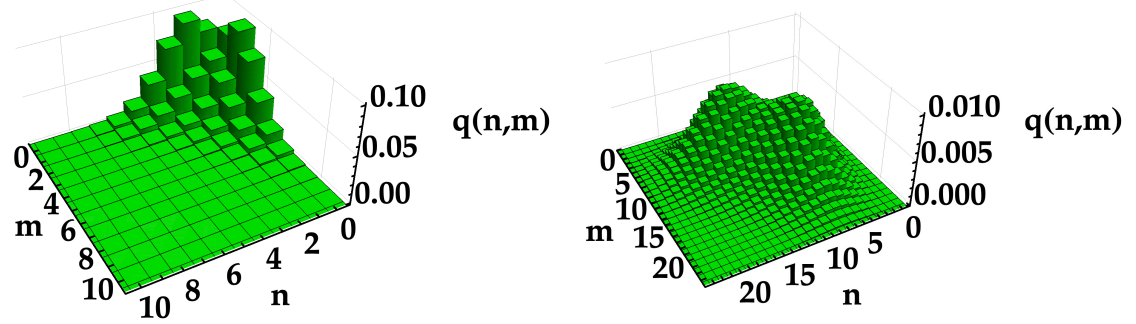

Figure 13: Joint-photon-number distribution in the case of Fock states $|2\rangle$ for $\eta=0.4$ and different choices of the LO intensity. Left: $|\beta|^{2}=5$, Right: $|\beta|^{2}=50$.

a detection efficiency $\eta=0.4$. In Fig. 13 we compare the joint probability distributions. As already noticed in the case of the single-photon Fock state, also for $|2\rangle$ the two peaks are almost superimposed because of the low quantum efficiency. At the same time, the homodyne-like distributions in Fig. 14 do not exhibit nothing more than one large peak with a small dip on the top.

It is interesting to notice the appearance of more than one non null contribution in the density matrix of Fig. 15, due to the low quantum efficiency. Moreover, the height of the non null contributions of the diagonal tends to the expected values at increasing intensity values of the LO. In this case, the maximum error on a single element of the density matrix is equal to 0.014 for $|\beta|^{2}=5$ and 0.010 for $|\beta|^{2}=50$, whereas the mean error on a single reconstructed element is equal to 0.004 for $|\beta|^{2}=5$ and 0.004 for $|\beta|^{2}=50$. The fidelities to the expected states are equal to $94.61 \pm 0.16 \%$ for $|\beta|^{2}=5$ and to $99.22 \pm 0.14 \%$ for $|\beta|^{2}=50$. The larger the photon-number state, the stricter the requirement 

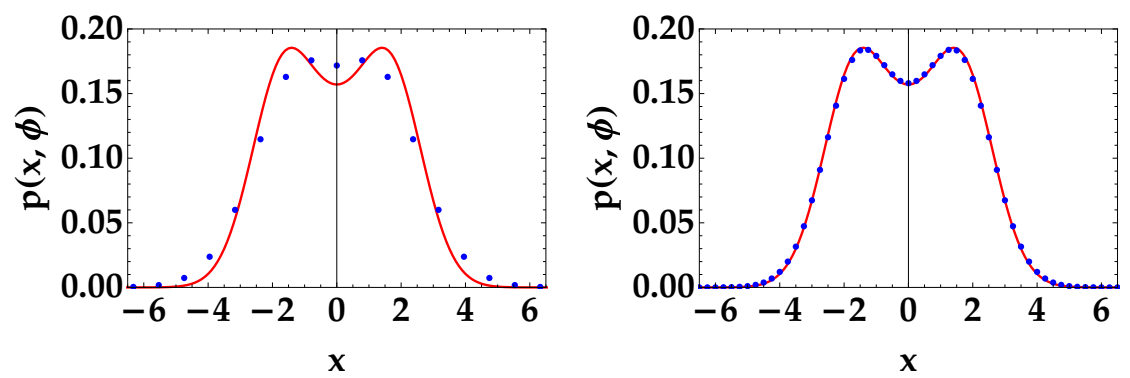

Figure 14: Homodyne-like distribution $p_{\mathrm{D}}$ in the case of Fock states $|2\rangle$ for $\eta=0.4$ and different choices of the LO intensity (blue dots). Left: $|\beta|^{2}=5$, Right: $|\beta|^{2}=50$. In each panel, the theoretical homodyne distribution is shown as red solid line. The fidelity between the two curves is equal to $99.322 \%$ for $|\beta|^{2}=5$ and $99.988 \%$ for $|\beta|^{2}=50$. In order to compare $p_{\mathrm{D}}$ and $p_{\mathrm{HD}}$, we suitably rescaled the photon-number difference $\Delta$ appearing in Eq. (4).
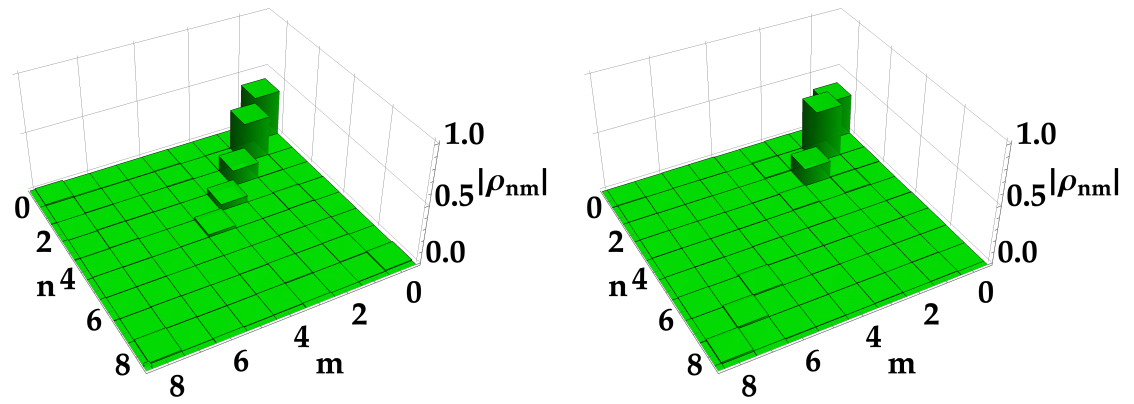

Figure 15: Reconstruction of the density matrix in the case of Fock states $|2\rangle$ for $\eta=0.4$ and different choices of the LO intensity. Left: $|\beta|^{2}=5$, Right: $|\beta|^{2}=50$.

on the LO, as also quantified by the state-fidelity values shown in Fig. 16 as a function of the LO intensity. It is worth noting that for the Fock state $|2\rangle$ the closeness of the reconstructed state to the expected one is slightly worse than for $|1\rangle$. This result can be ascribed to the fact that the increase of the intensity of the Fock state requires a more populated LO.

\section{Conclusions}

In this paper, we have investigated the role of the LO used in a homodynelike scheme for optical state tomography. In particular, we have tested how its intensity affects the different steps of the applied procedure in the case of Fock states $|1\rangle$ and $|2\rangle$, obtained by Monte-Carlo simulations. In general, we 


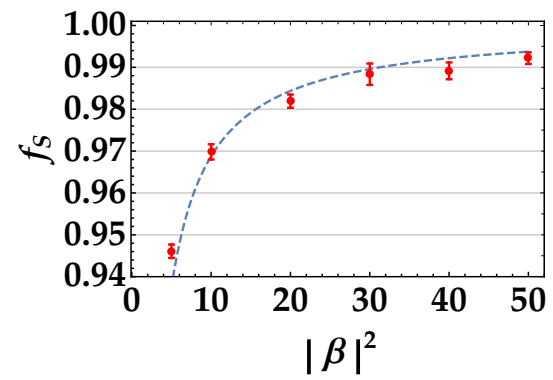

Figure 16: State fidelity in the case of Fock state $|2\rangle$ as a function of the LO intensity for $\eta=0.4$. The dashed line corresponds to the fitting function $g_{a}\left(|\beta|^{2}\right)$ with $a=0.312 \pm 0.023$.

can conclude that even a small imbalance between the signal state and the LO allows a reliable state reconstruction, at variance with the standard homodyne detection. However, the higher the intensity of the LO the better the reconstruction, as testified by the state fidelity, which exhibits an inverse dependence on the intensity of the LO. The actual value of intensity of the LO depends on the particular state under investigation and, more in detail, it depends on the behavior of its Wigner function and, thus, of its quadrature probability distributions. This result can be understood considering the general expression reported in Eq. (3).

The simulations are performed both in the case of an ideal detection efficiency, i.e. $\eta=1$, and in the case of a non-ideal one. In particular, we considered $\eta=0.4$, since it represents a reliable value of the quantum efficiency of commercial photon-number-resolving detectors, such as hybrid photodetectors [29] and silicon photomultipliers 22. It is interesting to notice that, even in this non-ideal condition, in which not only the signal state but also the LO is reduced, the reconstructed states resemble the theoretical expectation. All these results lead us to emphasize that the macroscopic nature of the LO in standard homodyne detection is not necessary for the state reconstruction, but it is required by the presence, in the scheme, of detectors operating in the macroscopic regime, i.e. the p-i-n photodiodes. Remarkably, a strong LO is also a way to avoid drawbacks from the detector dark current noise and, for instance, allows the quantum analysis of very weak entangled beams [30]. On the contrary, the 
use of a low-intensity LO and of photon-number-resolving detectors gives us the opportunity to investigate the role of the LO intensity and to better understand the real limitations in the reconstruction procedure. Thanks to its hybrid nature, the homodyne-like detection scheme can find applications in a Quantum Communication context exploiting both continuous- and discrete-variable states. In this case, one should switch between two quite different detection setups, namely standard homodyne detection and photon counting. While it is not possible requiring photon-number-resolving power to a homodyne detector, the homodyne-like configuration allows us to retrieve information both about the photon-number statistics and the quadrature field. This can be useful, for instance, for applications to Quantum Key Distribution [31 and to state discrimination with coherent [32] or squeezed [33] states of light.

\section{Acknowledgements}

Fruitful discussions with Matteo G. A. Paris and Giovanni Caiazzo are acknowledged.

\section{References}

[1] U. Leonhardt, Measuring the Quantum State of Light, Cambridge Univ. Press, Cambridge, 1997.

[2] A. I. Lvovsky, M. G. Raymer, Continuous-variable optical quantum state tomography, Rev. Mod. Phys. 81 (2009) 299.

[3] Z. Hradil, Quantum-state estimation, Phys. Rev. A 55 (1997) 1561 (R).

[4] A. Ourjoumtsev, R. Tualle-Brouri, P. Grangier, Quantum homodyne tomography of a two-photon Fock-state, Phys. Rev. Lett. 96 (2006) 213601.

[5] J. Řeháček, Z. Hradil, E. Knill, A. I. Lvovsky, Diluted maximum-likelihood algorithm for quantum tomography, Phys. Rev. A 75 (2007) 042108. 
[6] G. Puentes, J. S. Lundeen, M. P. A. Branderhorst, H. B. ColdenstrodtRonge, B. J. Smith, I. A. Walmsley, Bridging particle and wave sensitivity in a configurable detector of positive operator-valued measures, Phys. Rev. Lett. 102 (2009) 080404.

[7] A. Allevi, A. Andreoni, M. Bondani, G. Brida, M. Genovese, M. Gramegna, P. Traina, S. Olivares, M. G. A. Paris, G. Zambra, State reconstruction by on/off measurements, Phys. Rev. A 80 (2009) 022114.

[8] M. Esposito, F. Benatti, R. Floreanini, S. Olivares, F. Randi, K. Titimbo, M. Pividori, F. Novelli, F. Cilento, F. Parmigiani, D. Fausti, Pulsed homodyne Gaussian quantum tomography with low detection efficiency, New. J. Phys. 16 (2014) 043004.

[9] U. Leonhardt, M. Munroe, T. Kiss, Th. Richter, M. G. Raymer, Sampling of photon statistics and density matrix using homodyne detection, Opt. Comm. 127 (1996) 144.

[10] G. M. D'Ariano, M. G. A. Paris, Adaptive quantum homodyne tomography, Phys. Rev. A 60 (1999) 518.

[11] A. Zavatta, S. Viciani, M. Bellini, Quantum-to-classical transition with single-photon-added coherent states of light, Science 306 (2004) 660.

[12] D. Buono, G. Nocerino, V. D'Auria, A. Porzio, S. Olivares, M. G. A. Paris, Quantum characterization of bipartite Gaussian states, J. Opt. Soc. Am. B 27 (2010) A110.

[13] S. Cialdi, C. Porto, D. Cipriani, S. Olivares, M. G. A. Paris, Full quantum state reconstruction of symmetric two-mode squeezed thermal states via spectral homodyne detection and a state-balancing detector, Phys. Rev. A 93 (2016) 043805.

255 [14] S. Olivares, S. Cialdi, M. G. A. Paris, Homodyning the $\mathrm{g}(2)(0)$ of Gaussian states, Opt. Commun. 426 (2018) 547. 
[15] C. Porto, D. Rusca, S. Cialdi, A. Crespi, R. Osellame, D. Tamascelli, S. Olivares, M. G. A. Paris, Detection of squeezed light with glass-integrated technology embedded into a homodyne detector setup, J. Opt. Soc. Am. B 35 (2018) 1596.

[16] T. Lipfert, J. Sperling, W. Vogel, Homodyne detection with on-off detector systems, Phys. Rev. A 92 (2015) 053835.

[17] J. Sperling, W. Vogel, G. S. Agarwal, Balanced homodyne detection with on-off detector systems: Observable nonclassicality criteria, Europhys. Lett. 109 (2015) 34001.

[18] S. Olivares, A. Allevi, G. Caiazzo, M. G. A. Paris, M. Bondani, Quantum tomography of light states by photon-number-resolving detectors, New J. Phys. 21 (2019) 103045.

[19] G. S. Thekkadath, D. S. Philips, J. F. F. Bulmer, W. R. Clements, A. Eckstein, B. A. Bell, J. Lugani, T. A. W. Wolterink, A. Lita, S. W. Nam, T. Gerrits, C. G. Wade, I. A. Walmsley, Tuning between photon-number and quadrature measurements with weak-field homodyne detection, quantph/1908.04765 (2019).

[20] R. Nehra, M. Eaton, C. González-Arciniegas, O. Pfister, Loss tolerant quantum state tomography by number-resolving measurements without approximate displacements, quant-ph/1911.00173 (2019).

[21] G. Chesi, L. Malinverno, A. Allevi, R. Santoro, M. Caccia, A. Martemiyanov, M. Bondani, Optimizing Silicon photomultipliers for Quantum Optics, Sci. Rep. 9 (2019) 7433.

[22] G. Chesi, L. Malinverno, A. Allevi, R. Santoro, M. Caccia, M. Bondani, Measuring nonclassicality with Silicon photomultipliers, Opt. Lett. 44 (2019) 1371. 
[23] A. Allevi, M. Bina, S. Olivares, M. Bondani, Homodyne-like detection scheme based on photon-number-resolving detectors, Int. J. Quantum Inform. 15 (2017) 1740016.

[24] S. L. Braunstein, Homodyne statistics, Phys. Rev. A 42 (1990) 474.

[25] M. Freyberger, K. Vogel, W. Schleich, From photon counts to quantum phase, Phys. Lett. A 176 (1993) 41.

[26] A. Mandarino, M. Bina, C. Porto, S. Cialdi, S. Olivares , M. G. A. Paris, Assessing the significance of fidelity as a figure of merit in quantum state reconstruction of discrete and continuous-variable systems, Phys. Rev. A 93 (2016) 062118.

[27] A. I. Lvovsky, H. Hansen, T. Aichele, O. Benson, J. Mlynek, S. Schiller, State Reconstruction of the Single-Photon FockState, Phys. Rev. Lett. 87 (2001) 050402.

[28] K. Laiho, M. Avenhaus, K. N. Cassemiro, Ch. Silberhorn, Direct probing of the Wigner function by time-multiplexed detection of photon statistics, New J. Phys. 11 (2009) 043012.

[29] A. Allevi, M. Bondani, Nonlinear and quantum optical properties and applications of intense twin-beams, Adv. Atom. Mol. Phys. 66 (2017) 49-110.

[30] G. Nocerino, D. Buono, A, Porzio, S. Solimeno, Survival of continuous variable entanglement over long distances, Phys. Scr. T153 (2013) 014049.

[31] M. Cattaneo, S. Olivares, M. G. A. Paris, Hybrid quantum key distribution using coherent states and photon-number-resolving detectors, Phys. Rev. A 98 (2018) 012333.

[32] M. Bina, A. Allevi, M. Bondani, S. Olivares, Homodyne-like detection for coherent state-discrimination in the presence of phase noise, Opt. Express 25 (2017) 10685. 
[33] G. Chesi, S. Olivares, M. G. A. Paris, Squeezing-enhanced phase-shift-keyed binary communication in noisy channels, Phys. Rev. A 97 (2018) 032315. 